\title{
Safety and efficacy of tenecteplase versus alteplase in acute coronary syndrome: a systematic review and meta-analysis of randomized trials
}

\author{
Alexandre Guillermin ${ }^{1}$, David Jun Yan ${ }^{1}$, Arnaud Perrier ${ }^{1,2}$, Christophe Marti ${ }^{2}$
}

\begin{abstract}
${ }^{1}$ Faculty of Medicine, University of Geneva, Geneva, Switzerland
${ }^{2}$ Division of Internal Medicine, Rehabilitation and Geriatrics, Geneva University Hospitals, Geneva, Switzerland
\end{abstract}

Submitted: 28 December 2015

Accepted: 13 February 2016

Arch Med Sci 2016; 12, 6: 1181-1187

DOI: 10.5114/aoms.2016.58929

Copyright @ 2016 Termedia \& Banach

\author{
Corresponding author: \\ Dr Christophe Marti \\ Division of Internal Medicine, \\ Rehabilitation and Geriatrics \\ Geneva University Hospitals \\ rue Gabrielle-Perret-Gentil 2 \\ $\mathrm{CH}-1211$ Geneva 14, \\ Switzerland \\ E-mail: christophe.marti@ \\ hcuge.ch
}

\begin{abstract}
Introduction: Alteplase and tenecteplase are two widely used thrombolytic agents and are both approved for the treatment of acute myocardial infarction. These two molecules have increased fibrin specificity compared with older thrombolytics but distinct pharmacokinetic properties and may differ in terms of risks and benefits. We decided to review the available evidence comparing the safety and efficacy of these two molecules in acute coronary syndrome (ACS) or pulmonary embolism (PE).

Material and methods: To compare the efficacy and safety profile of alteplase and tenecteplase, we systematically searched PubMed, Cochrane and Embase for randomized studies comparing weight-adjusted alteplase to weight-adjusted tenecteplase in patients with ACS or PE. The primary endpoint was the risk of major bleeding, and secondary endpoints were risk of intracranial haemorrhage $(\mathrm{ICH})$, vessel recanalization and 30-day mortality. Results: Three studies including 17,325 patients with ACS were included in a quantitative meta-analysis. No study compared alteplase to tenecteplase in acute PE. Tenecteplase was associated with a statistically significant reduction of the risk of major bleeding compared to alteplase ( $R R=0.79$; 95\% Cl: $0.69-0.90, p=0.0002)$. The risk of intracranial haemorrhage $(\mathrm{RR}=0.96 ; 95 \% \mathrm{Cl}: 0.71-1.31, p=0.82)$ and 30 -day mortality $(\mathrm{RR}=1.02$; $95 \% \mathrm{Cl}: 0.9-1.15)$ were similar in patients treated with alteplase or tenecteplase. No difference was observed in the rate of vessel recanalization.

Conclusions: The available evidence suggests that tenecteplase is associated with a reduced risk of major bleeding compared to alteplase in ACS without evidence of reduced efficacy. These results are however mainly dependent on a single study.
\end{abstract}

Key words: thrombolysis, alteplase, tenecteplase, systematic review, acute coronary syndrome.

\section{Introduction}

Acute pulmonary embolism and coronary artery disease (CAD) are two common conditions, and CAD is the single most common cause of death worldwide. Each year coronary artery disease causes over 7 million deaths worldwide, and is responsible for 1 of every 3 deaths in western populations [1]. 
The in-hospital mortality of ST-elevation myocardial infarction (STEMI) varies between $6 \%$ and $14 \%$ and has decreased over the years in parallel with the improvement of reperfusion strategies [2]. Although primary percutaneous coronary intervention $(\mathrm{PCl})$ is the preferred reperfusion strategy in patients with STEMI [3], thrombolytic therapy is recommended if primary $\mathrm{PCl}$ cannot be performed within 120 min or within 90 min in patients presenting early $(<2$ h) with a large infarct [1]. Similarly, thrombolytic therapy has been shown to be effective for patients with pulmonary embolism (PE) and haemodynamic failure [4-6] and might be beneficial for some patients with intermediate-risk PE [7]. Despite its beneficial impact on mortality, thrombolytic therapy is associated with an increased risk of bleeding complications. Major bleeding occurs in about $10 \%$ of patients receiving thrombolytic therapy and intracranial haemorrhage in about $1 \%[8,9]$. Due to this risk of adverse events, physicians are often reluctant to administer thrombolytic therapy, leading to possible under-utilization of this potentially life-saving treatment [10]. Various thrombolytic agents are now available, with an increased fibrin specificity compared to first generation thrombolytics such as streptokinase or urokinase [11]. In the Global Utilization of Streptokinase and Tissue plasminogen activator for occluded coronary arteries (GUSTO) trial, alteplase was associated with increased efficacy compared to streptokinase, but at the cost of an increased risk of intracranial haemorrhage [12].

Alteplase (t-PA) and tenecteplase (TNK) are the two most widely used plasminogen activators and are both approved for the treatment of acute myocardial infarction $[1,4]$. These two molecules have different pharmacodynamic and pharmacokinetic properties. Tenecteplase is a mutated form of alteplase with higher fibrin specificity and a longer half-life of elimination and is usually administered as a bolus infusion, whereas alteplase is usually administered as a 2-hour continuous infusion. Some indirect comparisons in patients with acute PE have suggested that tenecteplase could be associated with an increased risk of haemorrhagic complications [9]. Nevertheless, in a large double-blind randomized clinical trial comparing alteplase to tenecteplase in patients with acute myocardial infarction, single bolus tenecteplase was equivalent to alteplase in terms of 30-day mortality and was associated with a lower risk of non-cerebral bleeding [8].

In order to compare the safety and efficacy profile of these two molecules, we decided to systematically review and meta-analyse randomized trials comparing weight-adjusted tenecteplase to weight-adjusted alteplase for patients with acute PE or acute coronary syndrome (ACS).

\section{Material and methods}

Search strategy, study selection, data extraction and quality assessment were performed following a pre-established protocol.

\section{Search strategy}

Two investigators (AG, DJY) independently searched PubMed, Embase and the Cochrane library using the following key words: "(Tenecteplase OR alteplase OR thrombolysis OR thrombolytic therapy) AND (pulmonary embolism OR myocardial infarction OR acute coronary syndrome)". The full electronic search strategy is available in the appendix. The search was limited to human studies and articles with an abstract, with no time limit. Studies in English, French, Italian and Chinese were searched. Bibliographies of retrieved articles were manually checked for additional references.

\section{Study selection and data extraction}

We included randomized controlled trials comparing administration of tenecteplase (30-50 mg) to weight-adjusted alteplase (80$100 \mathrm{mg}$ ) in patients with acute PE or ACS. Acute pulmonary embolism was defined by the presence of suggestive symptoms for 2 weeks or less with confirmation of the diagnosis of pulmonary embolism by a validated method (pulmonary angiography, computed tomography angiography, high-probability pulmonary ventilation/perfusion scan, lower limb venous compression ultrasound positive for a proximal deep vein thrombosis in patients with a clinical suspicion of PE or necropsy). Myocardial infarction or acute coronary syndrome was defined according to the third universal definition of myocardial infarction [13] (rise of cardiac biomarker with at least one value above the $99^{\text {th }}$ percentile of the upper reference limit with at least one of the following criteria: symptoms of ischaemia, ECG changes indicative of ischaemia, development of pathologic $\mathrm{Q}$ waves in ECG, imaging evidence of a new loss of viable myocardium or new wall abnormality, finding of an intracoronary thrombus by angiography). Two investigators (AG, DJY) independently evaluated studies for possible inclusion. Non-relevant studies were excluded based on the title and abstract. For potentially relevant studies, the full text was obtained and two investigators (AG, DJY) independently assessed study eligibility and independently extracted the data on study design, inclusion criteria, patient characteristics, drug regimen, safety and efficacy outcomes. Disagreements were resolved by consensus or by discussion with a third investigator (CM). 


\section{Outcomes and measurements}

The primary safety outcome was major bleeding (defined according to the 2005 ISTH or GUSTO definition). The second safety outcome was intracranial haemorrhage. All-cause mortality and the rate of coronary reperfusion were analyzed as efficacy outcomes.

\section{Quality of data reporting}

The risk of bias of included studies was assessed using the Jadad score [14] evaluating the quality of randomization, blinding and attrition (score 0 to 5). Two investigators (AG/DJY) independently assessed the risk of bias.

\section{Data analysis and synthesis}

All analyses were performed according to the intention to treat principle. Pooled risk ratios (RR) were obtained after extraction of the $2 \times 2$ tables of included studies using the Mantel-Haenszel method. The significance level was set at 0.05 for all analyses. Statistical heterogeneity was measured by the $l^{2}$ statistic. A fixed effect model was used when statistical heterogeneity was deemed low $\left(R^{2}<25 \%\right)$, and a random effect model was used in other cases. Potential heterogeneity factors were explored by pre-specified subgroup analyses including the type of pathology (ACS vs. $P E)$. A sensitivity analysis was conducted to check the robustness of the pooled risk ratios by removing each study one by one. Moreover, a sensitivity analysis was performed to evaluate the impact of inclusion of the study by Cannon et al. [15] on treatment effect estimates. For statistically significant results, we pooled risk differences using the Mantel-Haenszel method and calculated numbers needed to treat (NNT) and numbers needed to harm $(\mathrm{NNH})$ by the inverse of the absolute risk reduction, both with 95\% confidence intervals $(\mathrm{Cl})$. Publication bias was assessed using inspection of the funnel plot. Analyses were performed using the Cochrane Review Manager software (RevMan 5.2.8 - The Nordic Cochrane Centre, The Cochrane Collaboration, 2013).

\section{Results}

\section{Study selection and characteristics}

We retrieved a total of 5,834 references, among which 1,924 duplicates were identified. Of the 3,910 remaining articles 3,895 were excluded based on the title and abstract (Figure 1). The full text was obtained for the remaining 15 articles. Of these, 7 were not original, 1 was not a randomized study and 3 did not report the relevant outcomes. The study flow chart is provided in Figure 1. Four studies [8, 15-17] including a total of 18,208 patients met the inclusion criteria. One study was Asian [16], two North-American [15, 17] and one multicentric [8]. All studies compared alteplase to tenecteplase in acute myocardial infarction (STEMI). No study comparing alteplase versus tenecteplase in pulmonary embolism was found. Three of the four studies $[8,16,17]$ compared alteplase and tenecteplase at equal doses (TNK: 30-50-mg single bolus weight-adjusted, t-PA: $15-\mathrm{mg}$ bolus, followed by a $0.75-\mathrm{mg} / \mathrm{kg}$ (up to 50-mg) infusion over $30 \mathrm{~min}$, followed by $0.50-\mathrm{mg} / \mathrm{kg}$ (up to $35-\mathrm{mg}$ ) infusion over $60 \mathrm{~min}$ ). In the remaining study [15], tenecteplase was randomly administered at doses of 30,40 or $50 \mathrm{mg}$. Therefore, this study was included in the qualitative review but excluded from the quantitative meta-analysis. The characteristics of the included studies are provided in Table I.

\section{Major bleeding}

Three studies $[8,16,17]$ including 17,325 patients reported on major bleeding. The reported incidence of major bleeding was 4.8\% (399/8651) in the tenecteplase group and 5.8\% (507/8674) in the alteplase group (Figure 2). Overall, the risk of major bleeding was lower in patients receiving tenecteplase $(\mathrm{RR}=0.79 ; 95 \% \mathrm{Cl}: 0.69-0.90, p=$ $0.0002)$. In terms of absolute risk difference, this corresponded to a $1 \%(95 \% \mathrm{Cl}: 1.0-2.0 \%)$ risk

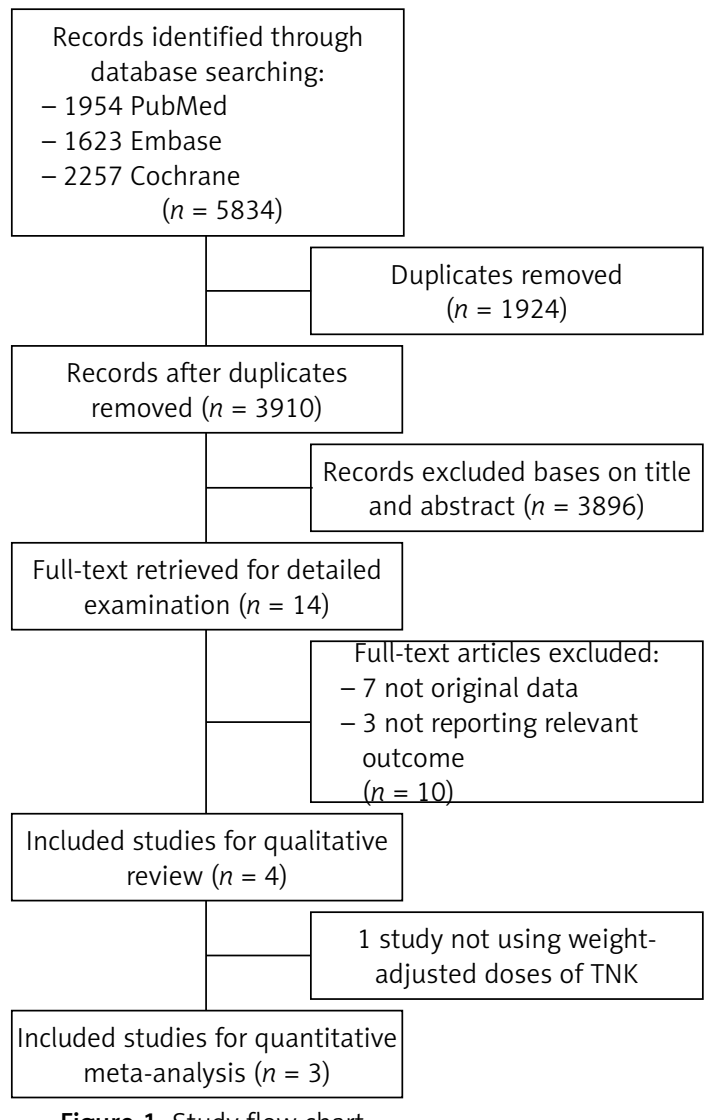

Figure 1. Study flow chart 


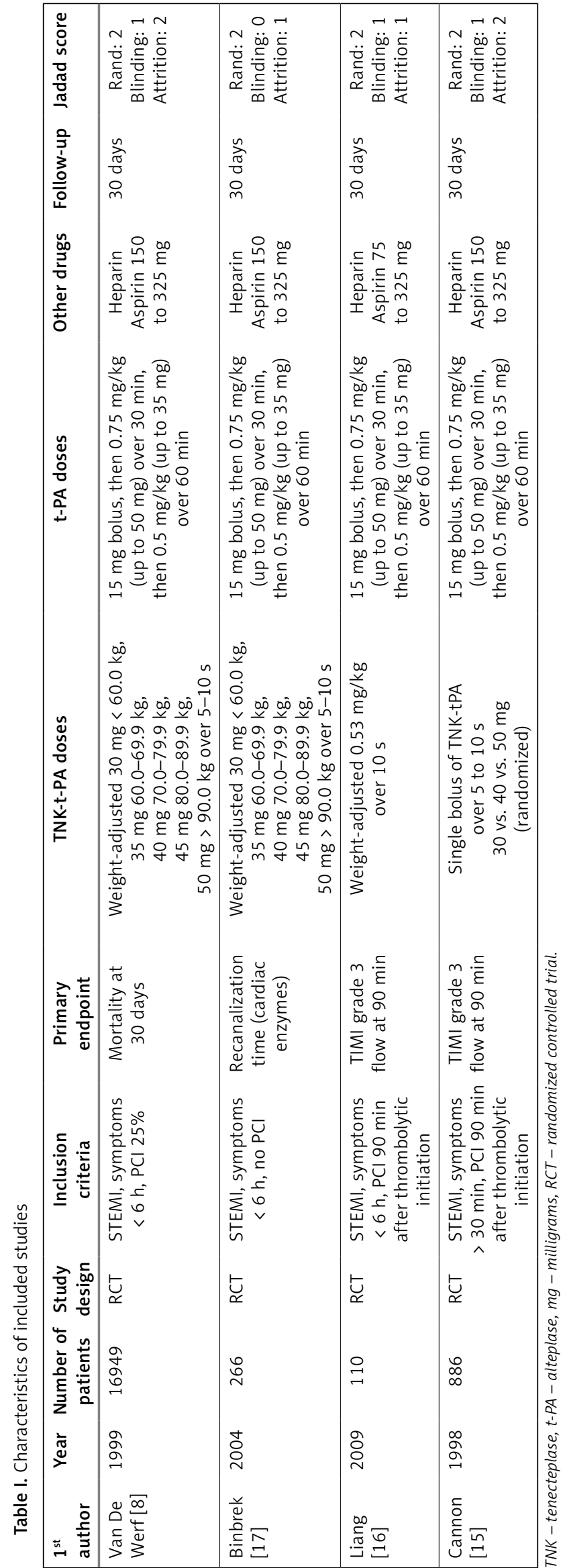

reduction of major bleeding (NNT 100; 95\% Cl: 50-100).

The Cannon study was not included in the meta-analysis since the dose of tenecteplase was not weight-adjusted in this study. Major bleeding occurred in $1.9 \%, 5.2 \%$ and $11.5 \%$ of patients treated with the 30-, 40-, and 50-mg doses of TNK-tPA, compared with $8.5 \%$ for tPA in this study.

\section{Intracranial haemorrhage}

Three studies $[8,16,17]$ including 17,325 patients reported on intracranial haemorrhage. The reported incidence of $\mathrm{ICH}$ was $0.91 \%$ (79/8651) in the tenecteplase group and $0.95 \%(82 / 8674)$ in the alteplase group (Figure 2). Overall, the risk of intracranial haemorrhage was similar in patients receiving weight-adjusted TNK-tPA or tPA ( $R R=0.96$; 95\% Cl: 0.71-1.31, $p=0.82$ ). In the Cannon study [15], the rates of ICH were $1.0 \%$ for $30 \mathrm{mg}$, $1.9 \%$ for $40 \mathrm{mg}$ and $3.8 \%$ for $50 \mathrm{mg}$ of TNK-tPA and $1.9 \%$ for tPA.

\section{Mortality within 30 days}

Three studies $[8,16,17]$ including 17,325 patients reported on mortality. The reported 30-day mortality was $6.2 \%(535 / 8651)$ in the tenecteplase group and $6.1 \%(528 / 8674)$ in the alteplase group (Figure 3). Overall, 30-day mortality was similar in patients receiving weight-adjusted TNK-tPA or tPA (RR $=1.02 ; 95 \% \mathrm{Cl}: 0.91-1.15)$. In the Cannon study [15], the rates of 30-day mortality were $3.6 \%$ for $30 \mathrm{mg}$ TNK-tPA, $6.5 \%$ for $40 \mathrm{mg}$ and $3.8 \%$ for $50 \mathrm{mg}$ compared to $5.7 \%$ for tPA.

\section{Reperfusion}

Three studies [15-17] reported on the rate of reperfusion. In the study by Liang et al. [16] TIMI grade flow 3 at 90 min was similar in patients treated with TNK-tPA (68.4\%) or tPA $(66.7 \%, p=1.00)$. In the study by Binbrek et al. [17], the time to recanalization was calculated according to the course of the appearance of creatine kinase isoforms and was significantly shorter in patients treated with TNK-tPA (208 $\pm 10 \mathrm{~min})$ compared to tPA (237 $\pm 9 \mathrm{~min}$ ), but the size of the infarct was similar. Finally, in the study by Cannon et al. [15], the rate of TIMI grade flow 3 at 90 min was $54.3 \%$ for $30-\mathrm{mg}$ TNK-tPA, $62.8 \%$ for $40-\mathrm{mg}$ TNK-tPA and $65.8 \%$ for the 50 -mg dose compared to $62.7 \%$ for tPA.

Since these studies used different definitions of reperfusion or different dosages of thrombolytics, a meta-analysis was not performed for this outcome.

\section{Heterogeneity and sensitivity analysis}

The analysis of heterogeneity was limited by the small number of included studies. No hetero- 


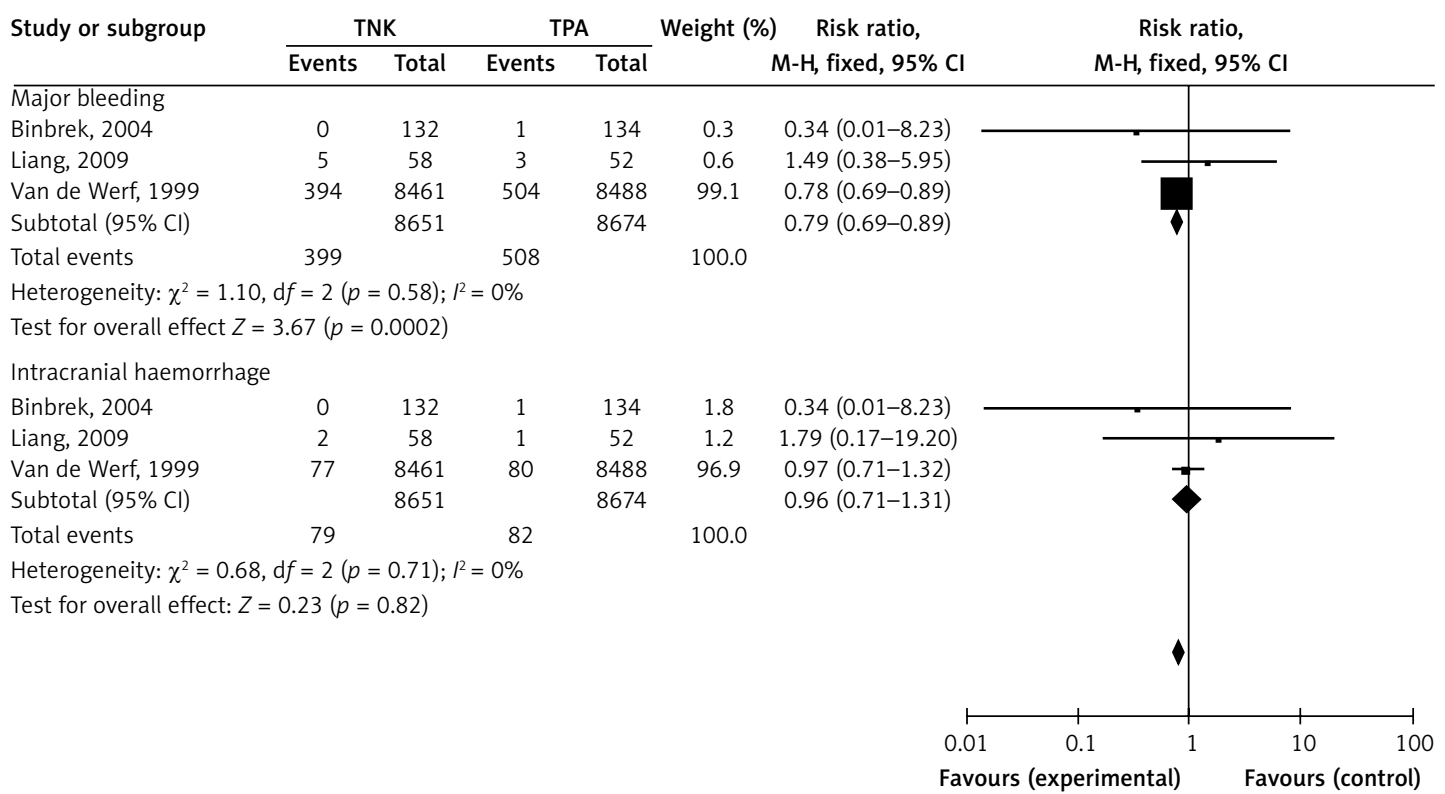

Figure 2. Major and intracranial haemorrhage

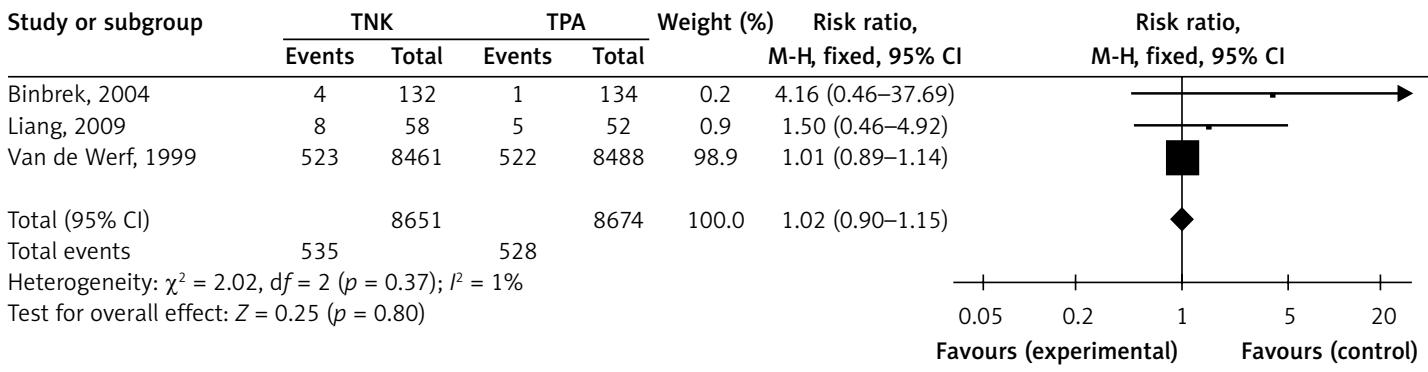

Figure 3. 30-day mortality

geneity was observed for any of the three main outcomes $\left(R^{2}=0-1 \%\right)$. Inclusion of the study by Cannon et al. [15] did not alter the estimation of our comparison for major bleeding $(\mathrm{OR}=0.76$; 95\% Cl: $0.66-0.86)$, mortality (OR $=1.00 ; 95 \%$ $\mathrm{Cl}: 0.89-1.13)$, or intracranial haemorrhage (OR = 0.94; 95\% Cl: 0.71-1.26). The difference in major bleeding was mainly dependent on the study by Van Der Werf et al. [8], and statistical significance was lost when this study was excluded (leaveone-out analysis). No changes in intracranial and mortality outcomes were observed after deletion of studies one by one. Evaluation of funnel plots (Figure 4) for potential publication bias was limited by the small number of studies.

\section{Discussion}

The available evidence suggests that weightadjusted TNK-tPA is associated with a reduced risk of major bleeding compared to tPA in patients with acute myocardial infarction and similar 30-day mortality. Although the benefit in major bleeding in our meta-analysis is mainly dependent on the ASSENT-2 trial, this study is a large

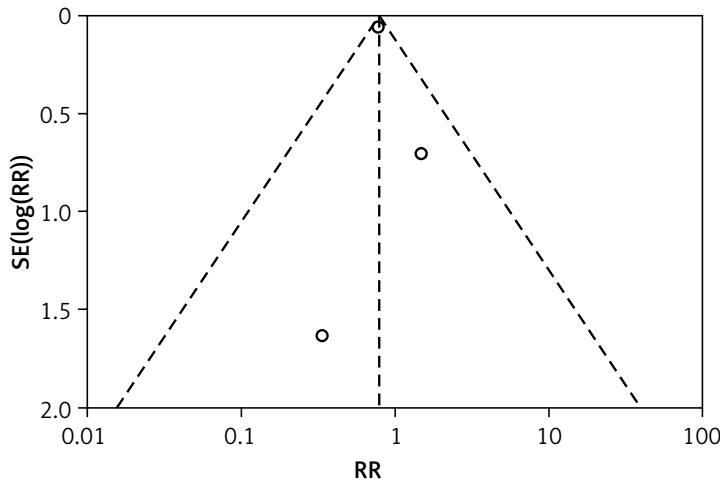

Figure 4. Relative risk of major bleeding according to study precision (funnel plot)

multi-centric trial with an estimated low risk of bias, and the observed difference in major bleeding seems unlikely to have happened by chance given the low $p$-value for this comparison. The results of the present meta-analysis contrast with previous publications. A systematic review about thrombolytic therapy for acute pulmonary embolism [9] reported a lower risk of major bleeding in patients receiving alteplase compared to those 
receiving tenecteplase. Nevertheless, these differences were probably due to the indirect nature of the comparison and the inclusion of older patients in studies using tenecteplase. Similarly, Mehta et al. [18] suggested an increased risk of intracranial haemorrhage with bolus versus infusion thrombolytic therapy among patients with acute coronary syndrome, but the seven studies included in their meta-analysis used six different bolus thrombolytic agents, repeated bolus administrations, and four different infusion comparators.

The benefit of bolus, weight-adjusted tenecteplase over weight-adjusted alteplase infusion observed in the present meta-analysis may be due to some pharmacokinetic advantages of tenecteplase over alteplase, including higher fibrin specificity, reduced binding to the physiological plasminogen activator inhibitor PAI-1 and prolonged plasma half life enabling single bolus administration [19]. Previous large comparative trials such as the GISSI-2 [20] or GUSTO-1 [12] trial reported fewer bleeding complications in patients treated with the more fibrin-specific agent alteplase compared to older, less fibrin-specific thrombolytics. More recently, a phase 2B randomized study comparing alteplase and tenecteplase [21] in patients with acute ischaemic stroke suggested superior efficacy of tenecteplase over alteplase in reperfusion and clinical improvement without an increase in adverse events. Nevertheless, no difference was observed in our meta-analysis regarding the risk of intracranial haemorrhage, but the confidence interval for this outcome was wide due to its low incidence, including a possible $30 \%$ between-group relative risk difference in favour of tenecteplase.

An alternative explanation for the observed reduction of major bleeding complications in patients treated with TNK could be the non-equivalence of the proposed regimens and doses of thrombolytics, leading to a lower thrombolytic potency of TNK. Unfortunately, the ASSENT-2 trial did not report on coronary reperfusion, and the overall mortality reflects both the risks and benefits of treatment arms. Nevertheless, the proportion of patients with complete recanalization was similar in the study by Liang et al., and the study by Binberk suggested a possible reduction of the time to recanalization among patients treated with TNK compared to tPA. Therefore, there is no evidence of reduced efficacy of TNK to explain the observed reduction of major bleeding.

The present work is to our knowledge the first systematic review of randomized studies allowing a direct comparison of the efficacy and safety TNK and TPA in patients with ACS. Despite the relatively small number of available original studies, the number of included patients was large enough to allow a precise estimation of treatment differences, at least for the safety outcomes. The results of our meta-analysis were mainly dependent on the ASSENT-2 study, and the analysis of potential heterogeneity factors among studies was limited. Nevertheless, the inclusion of the studies by Liang et al. [16] and Binbrek et al. [17] allowed a comparison of the thrombolytic efficacy of alteplase and tenecteplase, which was not evaluated in the ASSENT-2 study. Finally, all of the included studies were performed among patients with ACS, and it remains uncertain whether their results can be extrapolated to patients with acute PE, since these patients differ in terms of co-morbidities, demographics and concomitant use of anti-platelet therapy.

In conclusion, the available evidence suggests that weight-adjusted tenecteplase is superior to weight-adjusted alteplase in terms of safety, with a lower risk of major bleeding. More limited evidence suggests that tenecteplase and alteplase are equivalent in terms of efficacy.

\section{Acknowledgments}

Alexandre Guillermin and David Jun Yan equally contributed to this article

We thank Professor Mathieu Nendaz for revising the manuscript.

\section{Conflict of interests}

The authors declare no conflict of interest.

\section{References}

1. Steg PG, James SK, Atar D, et al. ESC Guidelines for the management of acute myocardial infarction in patients presenting with ST-segment elevation. Eur Heart J 2012; 33: 2569-619.

2. Van de Werf F, Bax J, Betriu A, et al. ESC guidelines on management of acute myocardial infarction in patients presenting with persistent ST-segment elevation. Rev Esp Cardiol 2009; 62: 293, e1-47.

3. Keeley EC, Boura JA, Grines CL. Primary angioplasty versus intravenous thrombolytic therapy for acute myocardial infarction: a quantitative review of 23 randomised trials. Lancet 2003; 361: 13-20.

4. Konstantinides S, Torbicki A, Agnelli G, et al. [2014 ESC Guidelines on the diagnosis and management of acute pulmonary embolism]. Kardiol Pol 2014; 72: 997-1053.

5. Jerjes-Sanchez C, Ramirez-Rivera A, de Lourdes Garcia M, et al. Streptokinase and heparin versus heparin alone in massive pulmonary embolism: a randomized controlled trial. J Thromb Thrombolysis 1995; 2: 227-9.

6. Duru S, Kelesoglu A, Ardic S. Clinical update on pulmonary embolism. Arch Med Sci 2014; 10: 557-65.

7. Meyer G, Vicaut E, Konstantinides SV. Fibrinolysis for intermediate-risk pulmonary embolism. N Engl J Med 2014; 371: 581-2.

8. Van De Werf F, Adgey J, Ardissino D, et al. Single-bolus tenecteplase compared with front-loaded alteplase in acute myocardial infarction: the ASSENT-2 double-blind randomised trial. Lancet 1999; 354: 716-22. 
9. Marti C, John G, Konstantinides S, et al. Systemic thrombolytic therapy for acute pulmonary embolism: a systematic review and meta-analysis. Eur Heart J 2015; 36: 605-14.

10. Stein PD, Matta F. Thrombolytic therapy in unstable patients with acute pulmonary embolism: saves lives but underused. Am J Med 2012; 125: 465-70.

11. Mueller HS, Rao AK, Forman SA. Thrombolysis in myocardial infarction (TIMI): comparative studies of coronary reperfusion and systemic fibrinogenolysis with two forms of recombinant tissue-type plasminogen activator. J Am Coll Cardiol 1987; 10: 479-90.

12. An international randomized trial comparing four thrombolytic strategies for acute myocardial infarction. The GUSTO investigators. N Engl J Med 1993; 329: 673-82.

13. Thygesen K, Alpert JS, Jaffe AS, Simoons ML, Chaitman BR, White HD. Third universal definition of myocardial infarction. Glob Heart 2012; 7: 275-95.

14. Jadad AR, Moore RA, Carroll D, et al. Assessing the quality of reports of randomized clinical trials: is blinding necessary? Control Clin Trials 1996; 17: 1-12.

15. Cannon CP, Gibson CM, McCabe CH, et al. TNK-tissue plasminogen activator compared with front-loaded alteplase in acute myocardial infarction: results of the TIMI 10B trial. Thrombolysis in Myocardial Infarction (TIMI) 10B Investigators. Circulation 1998; 98: 2805-14.

16. Liang F, Wang LZ, Hu DY, et al. [An angiographic trial to evaluate the efficacy and safety of tenecteplase in Chinese patients with acute myocardial infarction]. Zhonghua Xin Xue Guan Bing Za Zhi 2009; 37: 514-7.

17. Binbrek AS, Rao NS, Neimane D, Hatou E, Abdulali S, Sobel $\mathrm{BE}$. Comparison of rapidity of coronary recanalization in men with tenecteplase versus alteplase in acute myocardial infarction. Am J Cardiol 2004; 93: 1465-8.

18. Mehta SR, Eikelboom JW, Yusuf S. Risk of intracranial haemorrhage with bolus versus infusion thrombolytic therapy: a meta-analysis. Lancet 2000; 356: 449-54.

19. Tanswell P, Modi N, Combs D, Danays T. Pharmacokinetics and pharmacodynamics of tenecteplase in fibrinolytic therapy of acute myocardial infarction. Clin Pharmacokinet 2002; 41: 1229-45.

20. GISSI-2: a factorial randomised trial of alteplase versus streptokinase and heparin versus no heparin among 12,490 patients with acute myocardial infarction. Gruppo Italiano per lo Studio della Sopravvivenza nell'Infarto Miocardico. Lancet 1990; 336: 65-71.

21. Parsons M, Spratt N, Bivard A, et al. A randomized trial of tenecteplase versus alteplase for acute ischemic stroke. N Engl J Med 2012; 366: 1099-107. 